\title{
Gimbal-Less Monolithic Silicon Actuators for Tip-Tilt-Piston Micromirror Applications
}

\author{
Veljko Milanović, Member, IEEE, Gabriel A. Matus, and Daniel T. McCormick
}

\begin{abstract}
In this paper, fully monolithic silicon optical scanners are demonstrated with large static optical beam deflection. The main advantage of the scanners is their high speed of operation for both axes: namely, the actuators allow static two-axis rotation in addition to pistoning of a micromirror without the need for gimbals or specialized isolation technologies. The basic device is actuated by four orthogonally arranged vertical comb-drive rotators etched in the device layer of an silicon-on-insulator wafer, which are coupled by mechanical linkages and mechanical rotation transformers to a central micromirror. The transformers allow larger static rotations of the micromirror from the comb-drive stroke limited rotation of the actuators, with a magnification of up to $3 \times$ angle demonstrated. A variety of one-axis and two-axis devices have been successfully fabricated and tested, in all cases with $600-\mu \mathrm{m}$-diameter micromirrors. One-axis micromirrors achieve static optical beam deflections of $>20^{\circ}$ and peak-to-peak resonant scanning of $>50^{\circ}$ in one example at a resonant frequency of $4447 \mathrm{~Hz}$. Many two-axis devices utilizing four rotators were tested, and exhibit $>18^{\circ}$ of static optical deflection at $<150 \mathrm{~V}$, while their lowest resonant frequencies are above $4.5 \mathrm{kHz}$ for both axes. A device which utilizes only three bidirectional rotators for tip-tilt-piston actuation achieves $-10^{\circ}$ to $10^{\circ}$ of optical deflection in all axes, and exhibits minimum resonant frequencies of 4096 and $1890 \mathrm{~Hz}$ for rotation and pistoning, respectively. Finally, we discuss the preliminary results in scaling tip-tilt-piston devices down to $0.4 \times 0.4 \mathrm{~mm}$ on a side for high fill-factor optical phased arrays. These array elements include bonded low-inertia micromirrors which fully cover the actuators to achieve high fill-factor.
\end{abstract}

Index Terms-High aspect ratio, microfabrication, micromachining, micromirror, microoptoelectromechanical systems (MOEMS), optical microelectromechanical systems (MEMS), optical phased array, self-alignment, static optical deflection, tip-tilt-piston actuator, vertical comb drive.

\section{INTRODUCTION}

$\mathbf{T}$ HE silicon-on-insulator (SOI) technology for micromirrors and other optical components provides attractive features such as flat, smooth, and robust device layer, etch stop, CMOS compatibility, and relatively simple fabrication [1]-[10]. Previous static scanners in SOI have been limited to one axis and unidirectional rotation due to the electrically coupled lower comb fingers [4]-[6]. Recently, we have developed independently and linearly controllable vertical comb drives using only a single SOI device layer, thereby realizing monolithic and isolated structures [7], [8]. Our primary objective was to expand the technology to two-axis applications, while maintaining the high-speed operation achievable with one-axis devices. A fur-

Manuscript received October 23, 2003; revised March 23, 2004

The authors are with the Adriatic Research Institute, Berkeley, CA 94706

USA (e-mail: veljko@adriaticresearch.org).

Digital Object Identifier 10.1109/JSTQE.2004.829205 ther goal has been to achieve devices with tip-tilt-pistons, i.e. three degrees-of-freedom (DOF) actuation for phased array applications, which requires that the devices easily scale down to small dimensions.

Utilizing gimbaled structures is the most common method of implementing two-axis (2-DOF) rotation [1], [10], [11], although packaging-based methods are utilized as well. However, to implement 2-DOF gimbaled micromirrors without crosstalk between driving voltages, electrical isolation and mechanical coupling is necessary. Backfilling of isolation trenches by depositing an additional dielectric layer and chemical mechanical polishing (CMP) has been used to achieve the electrically isolated mechanical coupling [11], [12]. However, the additional deposition and CMP steps significantly increase complexity and cost. Another viable method is to leave part of the handle wafer unetched beneath the gimbal structure [10]. In all cases, complex fabrication has been required, and relatively low frequencies have been achieved due to the gimbals' slow outer axis. In applications where high-speed static scanning is required, the previous methods do not provide adequate solutions.

Our present approach, first proposed in [13], is to utilize recently demonstrated one-axis vertical comb drive-based rotation actuators [7], [8], which can by themselves achieve $>20^{\circ}$ of static optical deflection up to several kilohertz (with a $600-\mu \mathrm{m}$-diameter and $30-\mu \mathrm{m}$-thick silicon micromirror). They are discussed in Section II below. These rotators are then combined utilizing mechanical linkages that allow 2 DOF of rotation (tip-tilt,) or 3 DOF of motion (tip-tilt-piston) for a central micromirror. This is shown in Figs. 1, 2, and 3 and will be described in Section III. In such a way, we have decoupled the problem of two-axis scanners and can independently optimize and approach the problem of improving one-axis rotators, as well as the linkages that form the overall 2-DOF structure. While our current approach utilizes rotators to produce the actuation of the central micromirror, the design can also utilize pure vertical actuators or piston actuators [9].

In order to achieve the goal of large optical phased arrays with high fill factor (e.g. $>96 \%$ ), the actuators must be completely covered beneath the micromirror's reflecting plate for high fill factor; therefore, additional fabrication/packaging solutions are required. Some examples of previous work are given in [1], [14]-[17]. We currently address this issue by separately fabricating low-inertia silicon micromirrors and transferring/bonding them into place on top of the provided actuators stages. This is described in Section IV. The actuators must be reduced in size significantly to fit beneath a mirror with an edge dimension of less than $800 \mu \mathrm{m}$, while maintaining large torque and, therefore, high-speed operation. 
(a)

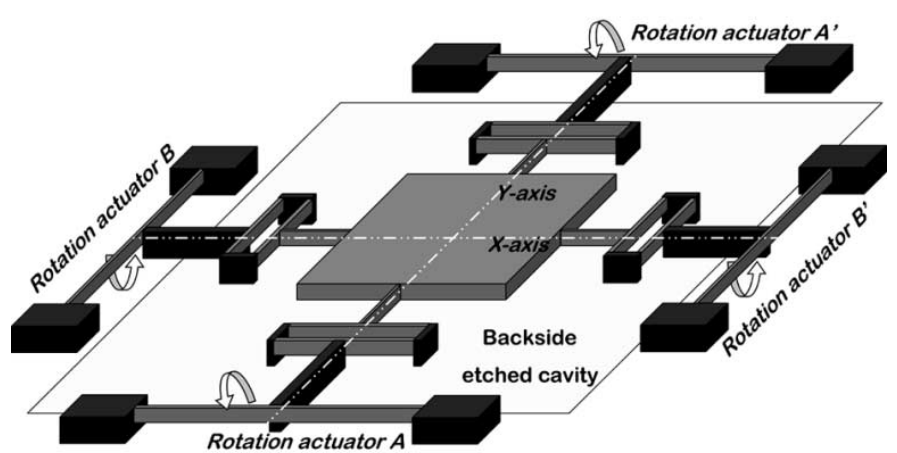

(b)

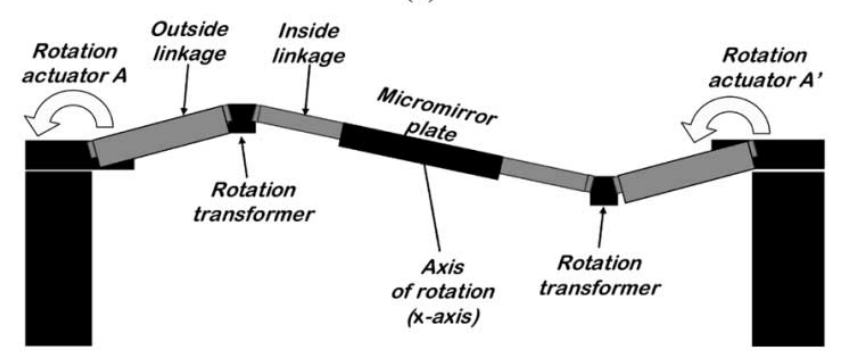

(c)

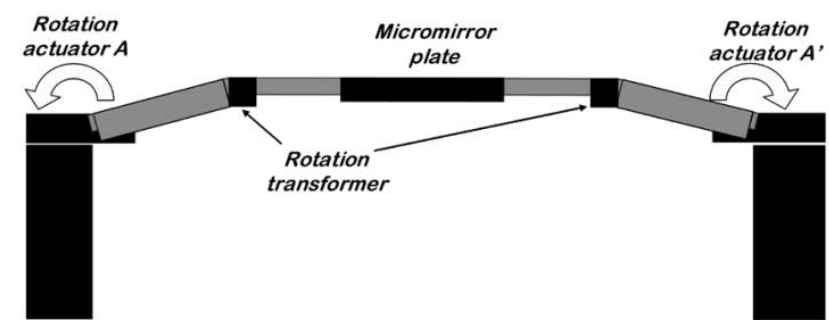

Fig. 1. Schematics of the scanners' operation. (a) Device consists of four vertical comb-drive rotation actuators, here shown just as torsion beams working in pairs to provide each of two axes of rotation. (b) Cross section A- $\mathrm{A}^{\prime}$ example of $x$-axis actuation, actuator $\mathrm{A}$ and $\mathrm{A}^{\prime}$ both turn in the same direction, giving micromirror rotation with virtual axis in center. (c) Same cross section with rotators $\mathrm{A}$ and $\mathrm{A}^{\prime}$ turning in opposite direction, in common-mode elevation of the transformers, giving vertical pistoning actuation.

In an array of such elements, routing of signals to each element must be provided. In the present work, we address actuator designs and micromirror bonding and transfer for high fill factor demonstration. The routing and packaging solutions for large arrays are planned as future work.

\section{BACKGROUND AND DEVICE PRINCIPLES}

\section{A. SOI Vertical Actuators}

Vertically staggered SOI comb drives perform well for single-sided rotation applications [4]-[6] and demonstrate the numerous advantages of SOI-microelectromechanical systems (MEMS) for optical applications. However, in these previous processes, no isolation is available between comb-drive fingers in either upper or lower comb drives, limiting devices to one-sided rotation. Rotation of devices is accompanied by undesired downward and lateral actuation due to the net electrostatic force, which is undesirable for many applications. Also, the support beams are full-thickness SOI device layer beams which are stiff for torsion-rotation and especially inadequate for pistoning. Lastly, the upper and lower comb finger sets
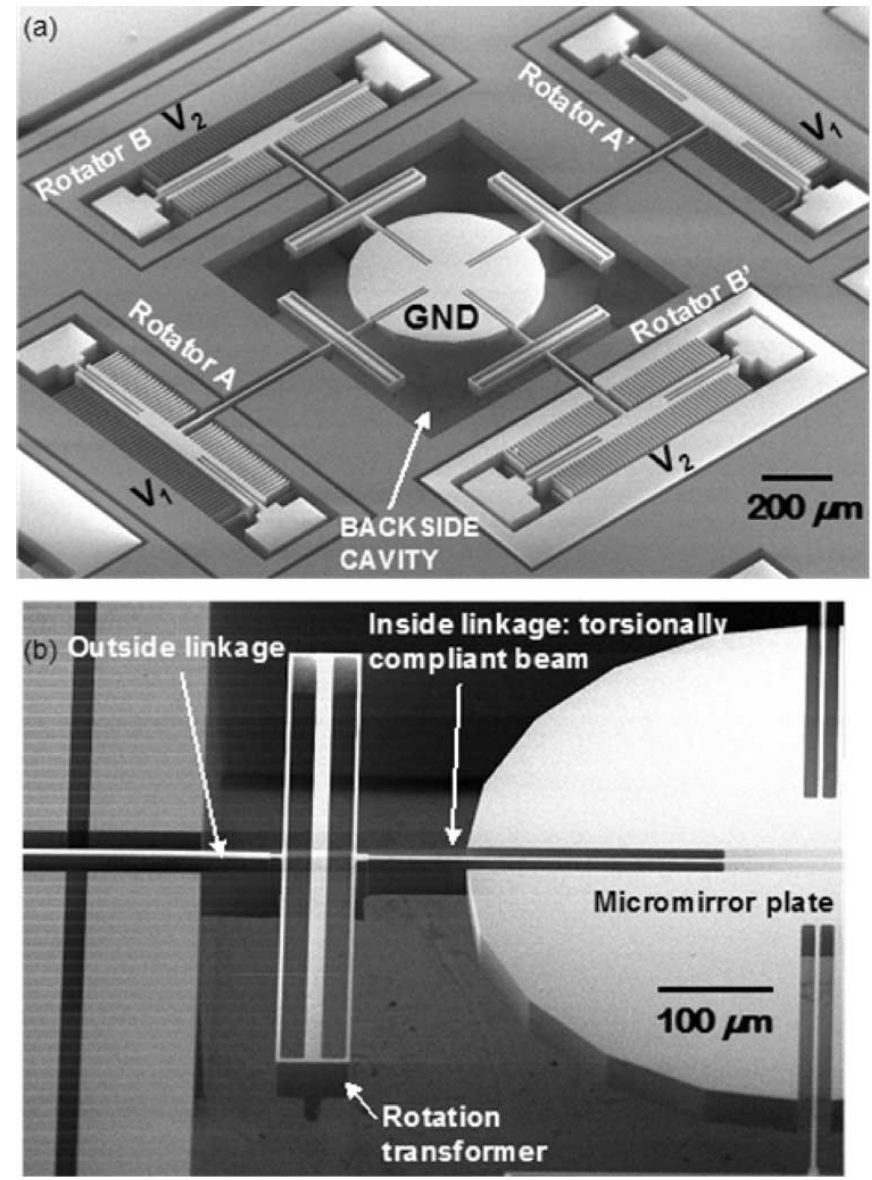

Fig. 2. SEM micrographs of a fabricated two-axis scanner. (a) Complete device with four actuators and a 600- $\mu$ m-diameter micromirror. (b) Close-up of one mechanical rotation transformer based on two parallel torsionally compliant beams.

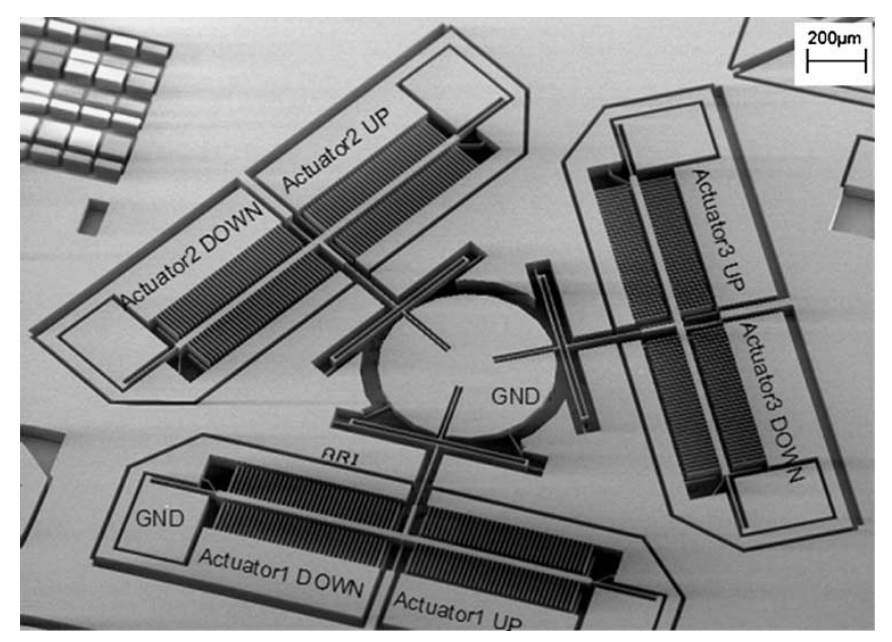

Fig. 3. SEM of a tip-tilt-piston device actuated by three bidirectional "pure" rotators which are arranged at $120^{\circ}$ to allow $3 \mathrm{DOF}$ (tip-tilt-piston) for the center micromirror. This device's lowest resonant frequencies are 4096 and $1890 \mathrm{~Hz}$ for rotation and pistoning modes, respectively.

are separated by the thickness of insulating oxide $(\sim 1 \mu \mathrm{m})$, sometimes requiring large biasing (pretilting) of devices before the comb fingers are adequately engaged. Preengagement of vertical comb fingers is desirable for well-behaved performance at lower actuation voltages [18]. This was previously 


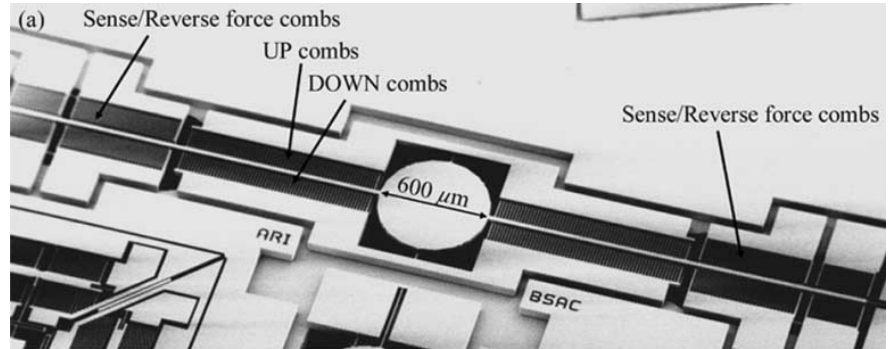

(b)
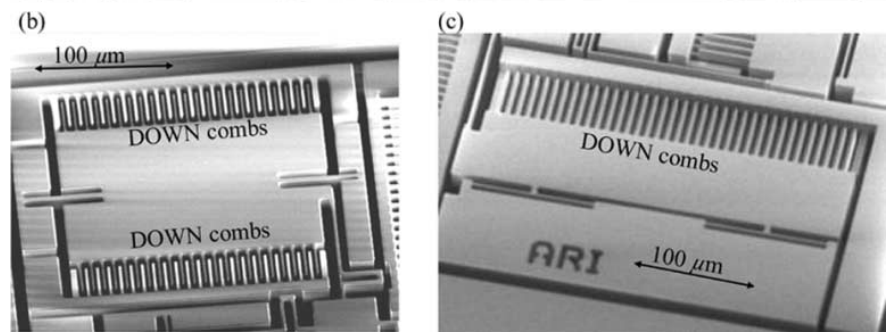

Fig. 4. Uni- and bidirectional one-axis rotators, which are the main building blocks of two-axis scanners and tip-tilt-piston actuators. (a) One-axis pure-rotation with integrated and isolated sense capacitance/reverse force actuation fingers. (b) Bidirectional Down rotation actuator. (c) Unidirectional Down actuator
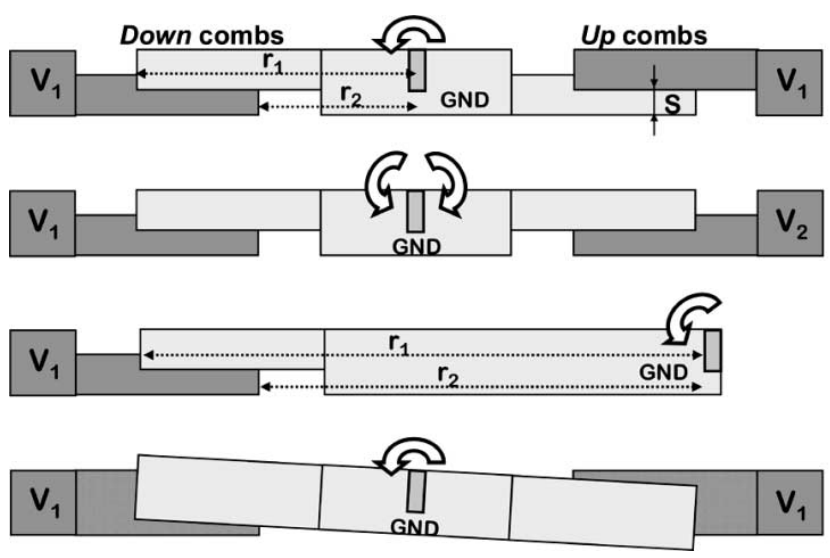

Fig. 5. Schematic cross sections of SOI device layer arranged to achieve four distinct modes of operation. (i) Pure unidirectional rotation. (ii) Bidirectional rotation and down pistoning. (iii) Unidirectional rotation. (iv) Reverse force actuation or sense capacitance fingers.

demonstrated in a silicon optical scanner fabricated by eutectic bonding assembly [19], as well as in the electrostatic vertical comb actuator fabricated in the BELST II process [20]. The process in [19] suffers from difficult alignment between comb fingers and utilizes metals and alloys that can reduce repeatability and reliability of device operation. The devices in [20] have relatively large spacing between successive comb fingers due to the need for specialized processing of the lower, stationary fingers and, therefore, reduced torque. In addition, release holes must be properly placed in the design, increasing design complexity and compromising the mirror's reflecting surface. The fabrication process utilized in this work, and detailed elsewhere [7], is a four-mask process that alleviates the above limitations, allowing various comb finger arrangements, as depicted in scanning electron microscopy (SEM) micrographs of Fig. 4 and their corresponding schematic cross sections of Fig. 5.
1) All comb fingers are fabricated in the device layer allowing isolated independently powered vertical comb-drive sets. This enables independent up or down pistoning and bidirectional rotation (Fig. 5). Those can be combined to achieve pure rotation, i.e. rotation with no lateral or vertical motion of the shuttle.

2) Comb fingers are timed etched such that there is several microns of preengagement (overlap), giving significantly better performance at lower voltages.

3) Support beams can be of any desired thickness for lower-voltage operation, and optimized rotation versus pistoning compliance.

4) Masks for etching of comb fingers are self-aligned by a single mask before any deep reactive ion etching (DRIE) steps.

5) Structures are made in monolithic single-crystal silicon for repeatable and reliable operation.

\section{B. Summary of Multilevel Beam SOI-MEMS Fabrication}

The process is described in detail in [7], and will be summarized here. The fabrication requires four photolithography masks - three for the desired three-level beams as shown in Fig. 6, and one for the bulk backside etch. The latter Backside mask provides dry release for devices in the SOI device layer, as well as space for rotation and vertical displacement of structures and integrated micromirrors. For that reason, the Backside mask is not necessary for small tip-tilt-piston array elements with bonded micromirrors, with minor modifications to the rest of the process.

1) SOI Wafer Preparation: The process begins by fabricating the 4-in SOI wafers. One wafer, intended for the SOI handle, is double-side-polished with a thickness of $300 \pm 1 \mu \mathrm{m}$. The second wafer which is to become the device layer, is an $n$-type wafer, standard thickness $(525 \pm 25) \mu \mathrm{m}$, and single-side polished. A wet thermal oxide of $1 \mu \mathrm{m}$ is grown on both wafers. The oxide on the handle wafer's side intended for bonding is patterned before the bonding: namely, after thermal oxide of $1 \mu \mathrm{m}$ was grown on both wafers, the wafer intended for SOI handle is patterned with mask Backup [Fig. 6(a)] and the oxide is etched down to silicon. After removing the photoresist mask and thorough cleaning, the wafers are prebonded, annealed, and sent for grinding and polishing to desired device layer thickness.

2) Mask Preparation and Self-Alignment Methodology: On the finished SOI wafers, the two front-side masks are prepared utilizing oxides of two thicknesses. The mask preparation is arranged to provide self-alignment of both front-side masks for high-performance vertical comb drives. In addition, due to the fact that the Backup mask is already buried within the SOI wafer, the mask preparation process requires that both of the front-side masks be aligned to that buried layer. These steps are given in detail in [7].

On the backside of the wafer, a single mask is employed and aligned to the front-side features. This, fourth Backside mask is applied with thick resist. Because the backside of the wafer also has $1.5 \mu \mathrm{m}$ of oxide from front-side preparation, the oxide is etched to Si substrate, and the wafer is prepared for DRIE steps as shown in Fig. 6(a). 
(a)

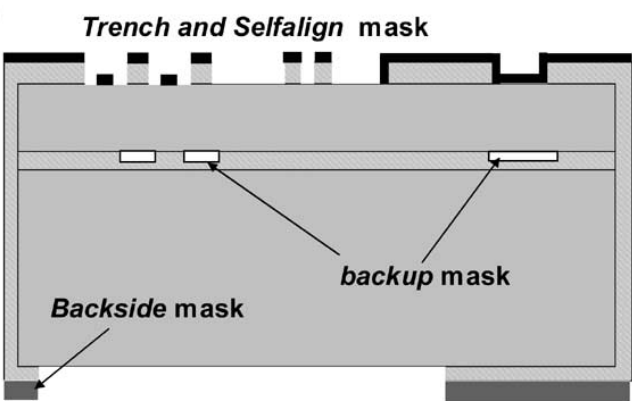

(b)

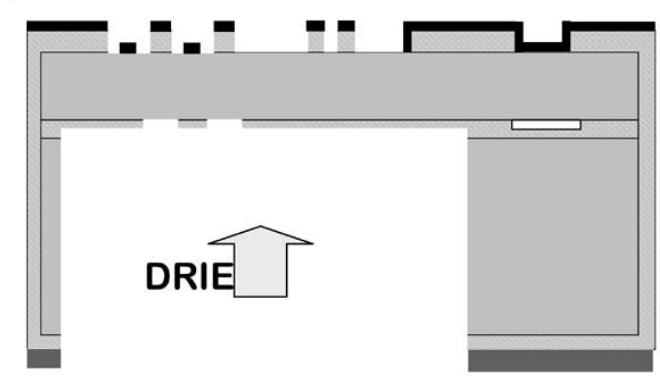

(c)

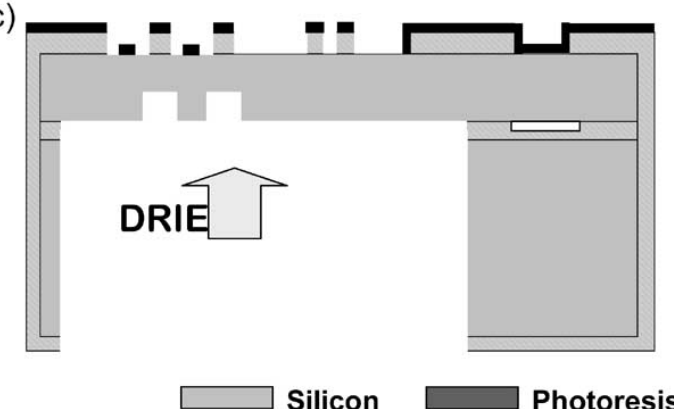

(d)
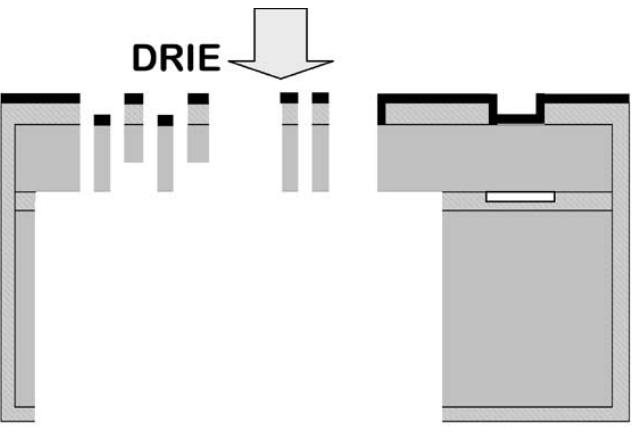

(e)

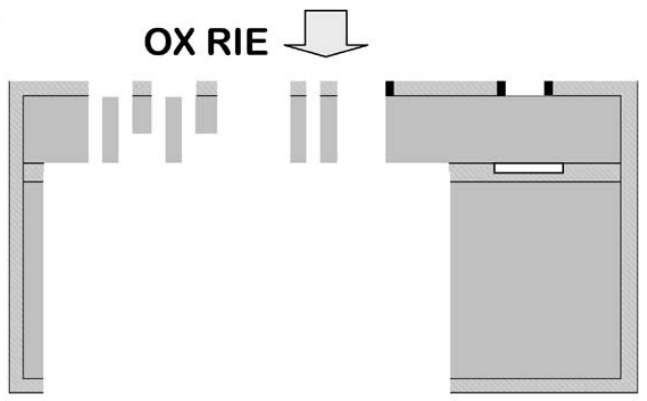

(f)

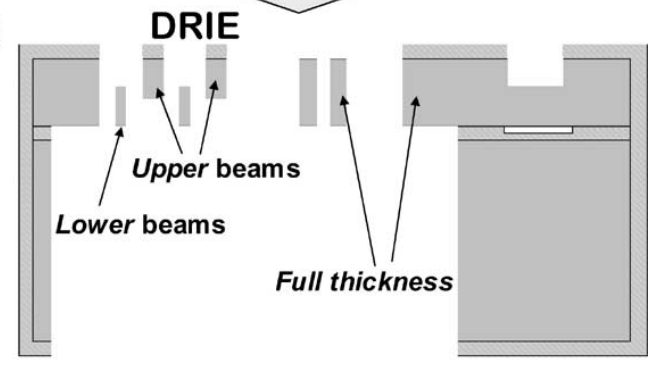

LTO

Wet ox.

Fig. 6. (a)-(f) Schematic of the etching process steps for the multilevel beam SOI-MEMS fabrication [7], utilized to fabricate the devices in this work. In (a), all four masks are already applied.

3) Backside DRIE: Backside etch process consists of multiple etches, as illustrated in Fig. 6(b) and (c). First, DRIE is done until the etched trench reaches the insulating oxide. This exposes the insulating oxide and the buried Backup mask [Fig. 6(b)]. The insulating oxide is then thinned (by timed oxide etch) $\sim 1.2 \mu \mathrm{m}$ which exposes the device silicon layer in areas of buried Backup mask. The final backside DRIE step shown is to perform the actual Backup DRIE into the device layer. This etch is timed to leave a desired thickness of Upper beams. In most cases we etched about $20 \mu \mathrm{m}$ of device layer silicon such that the remaining Upper beam thickness would be $\sim 30 \mu \mathrm{m}$. Lastly, the insulating oxide is fully removed from the backside [Fig. 6(c)].

4) Front-Side DRIE: The front-side DRIE steps are shown in Fig. 6(d)-(f) to better understand the formation of vertical comb drives. First. DRIE etches through the device layer as shown in Fig. 6(d). Then, an oxide plasma etch of $\sim 0.8 \mu \mathrm{m}$ on the front side thins down the oxide everywhere, removing the thinner oxide mask [Fig. 6(e)]. The second and final DRIE is performed until the devices are done, i.e., until the Lower beams are lowered to the desired height of $30 \mu \mathrm{m}$. The final result is shown in the schematic in Fig. 6(f).

\section{One-Axis Pure-Rotation Actuator}

As described in the introduction, the basic building block for the two-axis scanner is a one-axis rotator with the comb finger cross section as depicted in Fig. 5(i). As seen in Figs. 4(a) and 5(i), each actuator is composed of opposing comb fingers such that up actuation on one side and down actuation on the other side results in pure torque and no net vertical or lateral force.

The overlap area of comb fingers on either side of the actuator [Fig. 5(i)] has opposing rates of area change in case of lateral or vertical translation. However, during rotation (i.e., in the desired mode), overlap area increases on both sides and creates a nonzero overall area change. Because the comb fingers are rectangular in their cross sections, and start with an initial overlap, as seen in Fig. 5(a), calculations of exact overlap areas during rotation are more difficult than in the case of traditional lateral comb drives or vertical comb drives in pistoning mode. However approximations can be made to describe the geometry. The 
differential area increase $d A$ can be approximated as a wedge (triangle) from the rotation axis to the rotor finger tip, less the wedge from the rotation axis to the stator finger tip. The desired quantity $d A / d \theta$ is calculated from those two triangle areas as follows:

$$
\begin{aligned}
d A & \approx \frac{1}{2} r_{1} \cdot r_{1} d \theta-\frac{1}{2} r_{2} \cdot r_{2} d \theta \\
\frac{d A}{d \theta} & \approx \frac{1}{2}\left(r_{1}^{2}-r_{2}^{2}\right)
\end{aligned}
$$

where $r_{1}$ is the distance of the rotor finger tip from the rotation axis, and $r_{2}$ is the distance of the stator finger tip from the rotation axis [Fig. 5(a)]. This approximation is good to within $\sim 5 \%$. Because the fingers are initially preengaged such that linear operation from onset can be assumed [18], we can make a further approximation, neglecting fringing field effects

$$
\frac{d C}{d \theta} \approx 2 \frac{\varepsilon_{0}}{g} \cdot \frac{d A}{d \theta} \approx \frac{\varepsilon_{0}}{g} \cdot\left(r_{1}^{2}-r_{2}^{2}\right)
$$

where $e_{0}$ is air permittivity and $g$ is the gap distance between opposing comb fingers. The factor 2 in (3) comes from the fact that a comb finger has two sides which contribute to the capacitance. We then plug (3) into the well-known torque equation

$$
\tau=N \frac{1}{2} \cdot \frac{d C}{d \theta} \cdot V^{2} \approx \frac{N \cdot \varepsilon_{0} \cdot V^{2}}{2 g} \cdot\left(r_{1}^{2}-r_{2}^{2}\right)
$$

accounting for $N$ comb fingers in an actuator. Due to the complex actuator geometry, a more precise numerical solution to $d A / d \theta$ should be used that accounts for the shape of the comb finger and offsets in rotation axes beyond this range. Fringing fields at the outer extents of the finger travel result in capacitance greater than expected by simple overlap area, making (2) less accurate.

This approximation is only valid in the range $0^{\circ}<\theta<$ $\theta_{\text {max_lin. }}$ Rotation is an out-of-plane motion and eventually causes the fingertips to disengage. As the comb finger tips pass through, the rate $d A / d \theta$ is no longer constant and the drive force no longer follows the voltage squared law. Comb finger thickness and comb finger length are the two main parameters that determine this disengagement angle. The simplest approximation for the maximum angle is thus simply $\theta_{\text {max_lin }} \approx \tan ^{-1}\left(S / r_{1}\right) \approx S / r_{1}$ [Fig. 5(a)-(i)]. The drive will still continue to rotate past $\theta_{\text {max_lin }}$; however, the rate $d A / d \theta$ decreases and eventually changes sign, i.e., the area begins to decrease and the vertical comb drive loses further drive ability. This determines the maximum amount of rotation $\theta_{\max }$. The increasing effect of fringing fields makes $d A / d \theta, \tau$, and even $\theta_{\max }$ difficult to calculate.

A tradeoff in designing these types of actuators is that available torque increases with length squared [equation (2)], but at the same time limits the maximum available angle linearly and increases the likelihood of pull-in instability. For negative angles, clockwise in Fig. 5(i), (3) continues to apply, but only for a very short range before the upper comb fingers rotate out of plane and disengage from the lower fingers. Beyond this point, $\tau$ is very small and is difficult to calculate due to the effect of fringe fields. Generally speaking, these actuators are not effective for angles less than $0^{\circ}$.

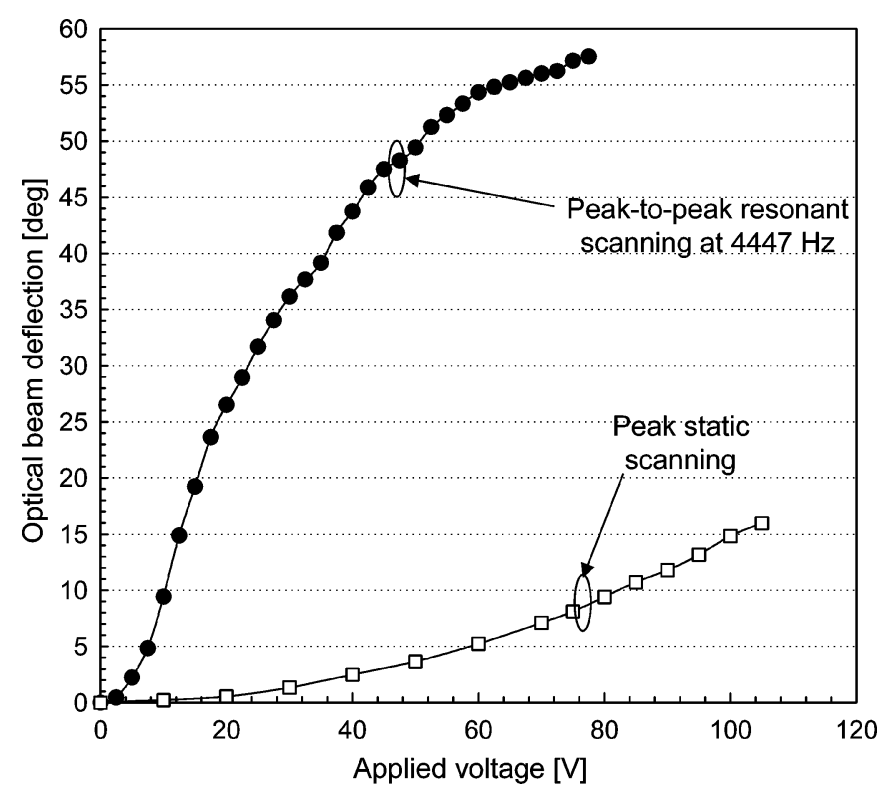

Fig. 7. One-axis micromirror with two opposing actuators combined with linkages as shown in Fig. 1(b) under static micromirror scanning and resonant scanning at $4447 \mathrm{~Hz}$.

It should be noted that the above discussion pertains only to static actuation of the devices, while their operation at the natural resonant frequency of the mechanical structure can be quite different, depending on the forcing waveform in the actuators. By choosing the proper pulsed forcing waveform, which allows the high-Q resonance to build up amplitude past the limitations given above as $S / r_{1}$, very large peak-to-peak amplitudes can be achieved. In one device, discussed below in Section II-D, over $55^{\circ}$ peak-to-peak optical deflection is shown in the data of Fig. 7. This was done by applying a pulsed waveform with $54 \%$ duty cycle at $4447 \mathrm{~Hz}$, which was previously determined to be the resonant frequency of the device.

\section{One-Axis Micromirror With Two Opposing Rotating Actuators}

As a step toward implementing gimbal-less two-axis devices, we tested one-axis devices which operate based on the methodology shown in the cross-sectional schematic of Fig. 1(b): namely, two rotating actuators are placed in parallel and arranged to rotate in the same [counterclockwise-Fig. 1(b)] directions. However, as their outside linkages and inside linkages connect between them to a micromirror plate, their rotation actually has opposing action. The rotator on the left actuates its outside linkage up, while the rotator on the right actuates down. As a result, the inside linkages effectively achieve rotation, in the opposite (clockwise) direction from the actuators. Test results for such a device are given in Fig. 7.

By the help of transformers and outside linkages, actuator rotation displaces the inside linkages in opposite directions and rotates the mirror clockwise. Since the outside and inside linkages experience the same vertical motion at the end of the transformer, the linkage rotation is inversely proportional to its length. Therefore, the ratio of actuator and mirror rotations can be scaled by changing the ratio of linkage lengths. As explained in Section II-C, vertical comb-drive stroke is limited by the device layer thickness. By scaling the linkage lengths, 
however, we can drive mirrors to rotation angles well beyond the rotational range of the actuators. This mechanical gain feature is explained in more detail in Section III below.

\section{E. Other Types of Actuators}

The type of actuator in Fig. 4(b), and schematically depicted in Fig. 5(ii), utilizes Down combs on both sides, resulting in bidirectional rotation when one side is activated at a time, though with some unwanted lateral and vertical motion. With both sides activated, it gives pistoning Down motion. The same approximations from (1)-(4) above apply, except that only one-half of the total number of comb fingers actuate in either direction.

The third type of actuator in Fig. 4(c), schematically depicted in Fig. 5(iii), utilizes only Down combs to provide unidirectional rotation. The tradeoff is that given the same space considerations as in Fig. 5(ii) and Fig. 5(i), the torque arm can be twice as long giving four times the torque.

Finally, the fourth type of actuator in Fig. 5(iv) utilizes full thickness, High beams for comb fingers which are perfectly overlapping at $\theta=0$. However, as $\theta$ increases beyond zero to either direction, applying voltage to this actuator gives reverse force, i.e. force to return to $\theta=0$. The second purpose for such comb drives is to utilize them as isolated sets of capacitance sense fingers, as in the device of Fig. 4(a). In that case, during the device operation, monitoring the capacitance on those comb drives provides position feedback for closed-loop feedback control.

Equations governing these types of comb fingers follow from above, approximating the area change and therefore torque as

$$
\tau \approx-\frac{N \cdot \varepsilon_{0}}{2 g} \cdot\left(r_{1}^{2}-r_{2}^{2}\right) \cdot V^{2}, \quad \theta \neq 0 .
$$

When used in capacitive sensing circuits, reference capacitors are fabricated on chip utilizing the same layout in order to insure matched capacitances.

\section{Design AND Characterization OF GimbaL-LesS TWO-AXIS SCANNERS}

As mentioned above in Section I, to achieve the goal of fast two-axis scanning, we desired to combine multiple one-axis rotators positioned orthogonally and utilize mechanical linkages to allow two axes of rotation for a central micromirror. The problem is how to combine one-axis scanners discussed above in Section II-D and allow their operation to be nearly independent of the other axis' operation. This proposed solution is schematically shown in Fig. 1 and a fabricated corresponding device in Fig. 2(a). Specifically, two one-axis rotators are utilized for each axis of the overall two-dimensional (2-D) scanner. For the $x$ axis, actuators $\mathrm{A}$ and $\mathrm{A}^{\prime}$ are utilized, and for the $y$ axis, actuators $\mathrm{B}$ and $\mathrm{B}^{\prime}$. The actuators are attached to the mirror through a set of linkages and a mechanical rotation transformer, as depicted in Fig. 1(b). The operation of a single axis was discussed above. It should be added that the inside linkages have to be designed such that they allow torsion on axis, specifically during the operation of the orthogonal axis. In other words, each linkage that connects a rotator to the central micromirror is actually designed to be a 2-DOF mechanical component which

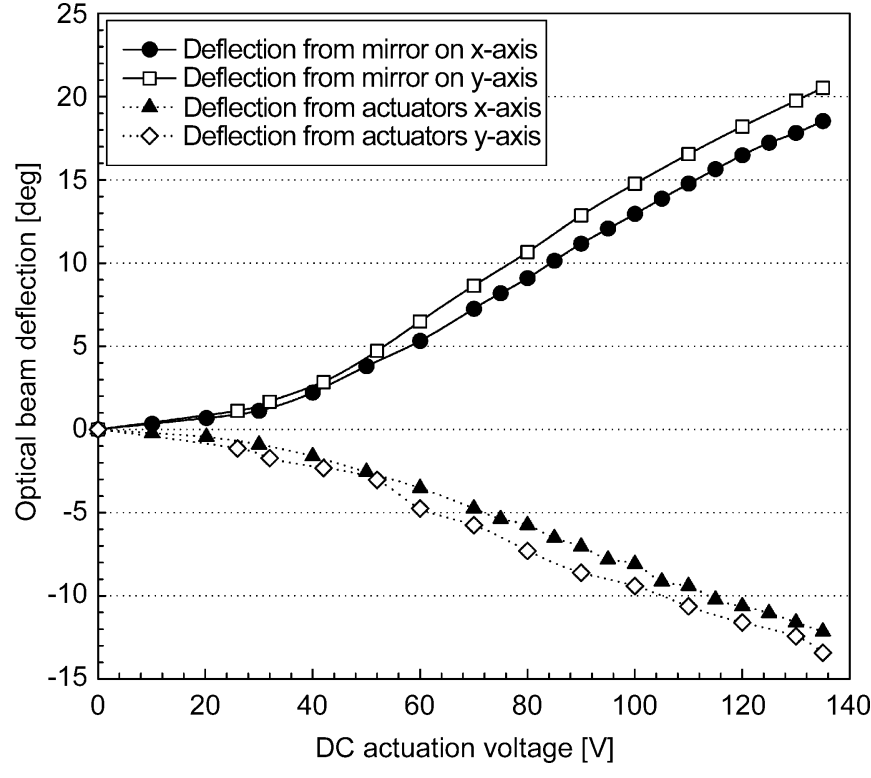

Fig. 8. Characterization of a device in static scanning. The measurements show both the deflections of the $600-\mu \mathrm{m}$ micromirror reflector as well as the deflections of the corresponding actuator pair (in opposite direction.) For both axes, a gain of $\sim 1.5$ is observed-for smaller actuator deflections, the mirror deflects $1.5 \times$ higher. The device's lowest resonant frequency for either axis is $4560 \mathrm{~Hz}$.

allows rotation via transformers and allows decoupling from the orthogonal axis via torsional compliance, usually in the inside linkage.

An important design feature is that the position of the mechanical rotation transformer in Figs. 1(b) and 2(b) governs the mechanical rotation gain $G$ between the micromirror's and actuator's rotation: namely, since the actuator rotates about its axis, and the micromirror stage rotates about a virtual stationary axis [due to opposing actuations on either side in Fig. 4(b)], the angles relate as $\theta_{\text {mirror }} / \theta_{\text {act }}=G=d_{2} / d_{1}$. In that, $d_{1}$ is the length of the inside linkage from the transformer to the mirrors center, and $d_{2}$ is the length of the outside linkage from the actuator's axis to the transformer. This feature is very beneficial due to the following: the maximum angle of each rotator is given by combdrive dimensions as discussed in Section II-C above, while the micromirror angle can be arbitrarily increased by using $G>1$. Moreover, proper design use of mechanical gain can significantly increase overall device speed as $G^{-1 / 2}$, because it allows increased finger length and torque while achieving same desired large angles of micromirror rotation.

A variety of designs have been designed and fabricated, in all cases utilizing 4 one-directional actuators arranged around the central mirror. Several devices of each kind were tested. By electrically activating the proper pair of electrodes, different actuation modes have been independently demonstrated. First, to characterize static deflection for each axis, the corresponding actuator pairs (A and $\mathrm{A}^{\prime}$ for the $x$ axis, and $\mathrm{B}^{2}$ and $\mathrm{B}^{\prime}$ for the $y$ axis) were used in common mode (same voltage applied.) By observing the deflection of a laser beam against a metric wall, we measured the rotations of both the micromirror as well as the actuators which reflect a small portion of the beam (the laser beam covers the entire device.) Static optical scanning measurements of one device are given in Fig. 8. For that device, lowest resonant frequency for both axes was $4560 \mathrm{~Hz}$. When 
(a)

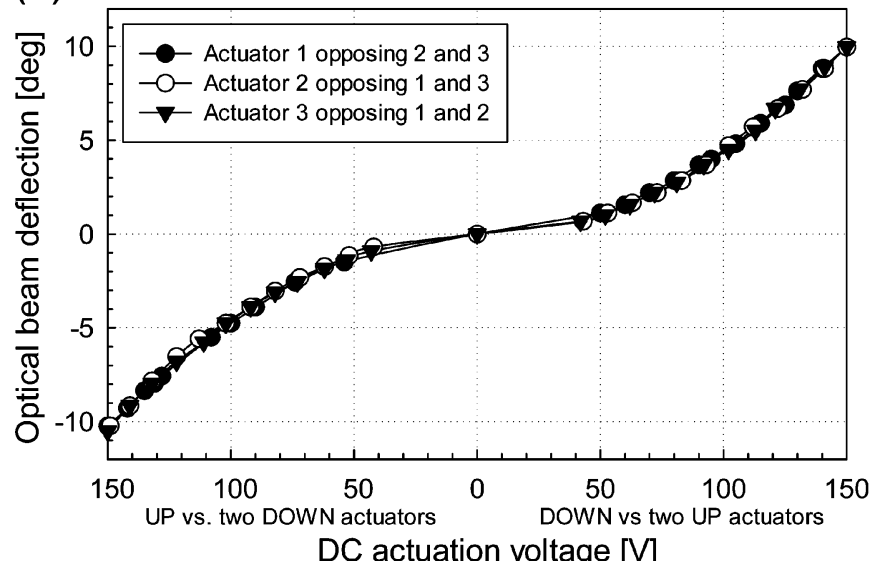

(b)

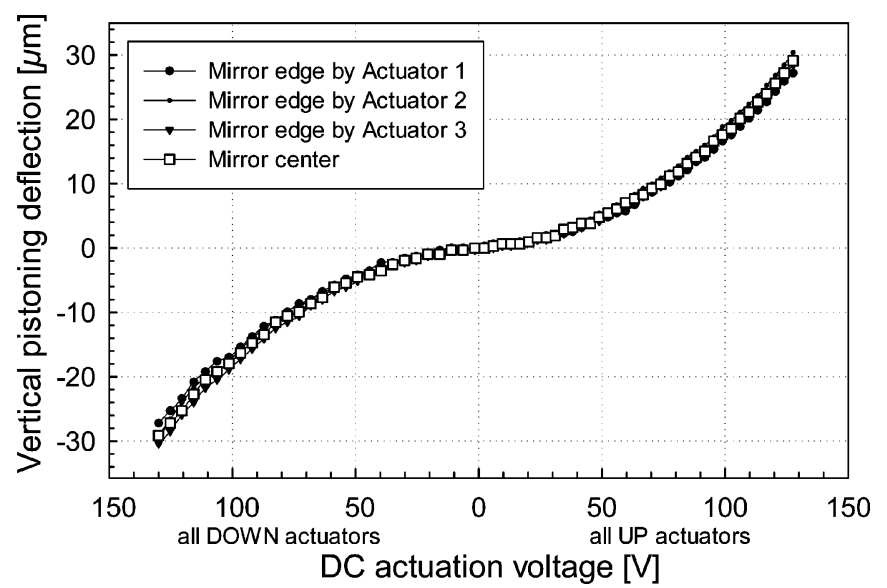

Fig. 9. Characterization of the three-actuator device in Fig. 3 in static scanning. Actuation voltages are all positive, however by choosing the proper set of actuators "negative" angle scanning is achieved.

running under resonance at that frequency, $40^{\circ}$ peak-to-peak optical deflection was observed with a pulsed waveform of $20-\mathrm{V}$ peak. In experiments where asymmetric voltages were applied to opposing actuators, pistoning effects were observed as well as rotation, though these modes are currently being further characterized.

The two-axis actuation is not in fact "optimized" in such a design: namely, instead of the four actuators placed at right angles, three actuators are sufficient to achieve efficient actuation, when arranged at $120^{\circ}$ to each other. This has a benefit of reduced area, but also a direct mechanical advantage. In such a "triangle" arrangement shown in Fig. 3, any inside linkage only rotates one-half of the angle of mirror rotation to allow decoupling. We have fabricated and tested devices with triangle arrangements, although they require more involved control due to the required mapping between the three axes and the more commonly used Cartesian $x, y$ coordinate system. Measurements for the device in Fig. 3 are given in Fig. 9. It was tested for three different axes: namely, the device shown in Fig. 3 has three bidirectional pure-rotation actuators, such that any of the actuators can either actuate the linkages up or down, depending on which electrode is activated. For testing of the "actuator 1" axis in the + angle direction, for example, we actuate the "actuator 1" down while actuating actuators 2 and 3 up. The actual axis of that rotation is (a)

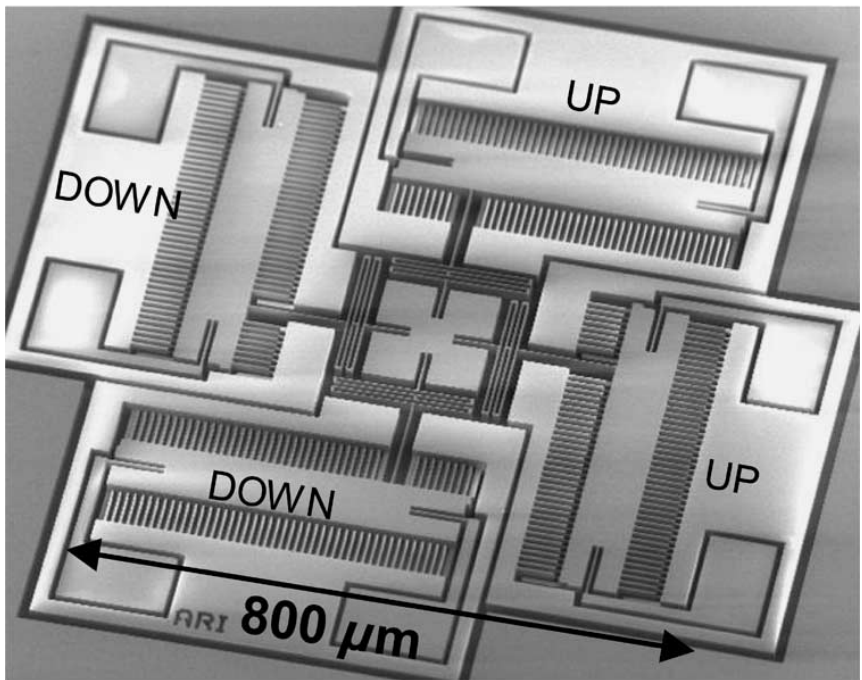

(b)

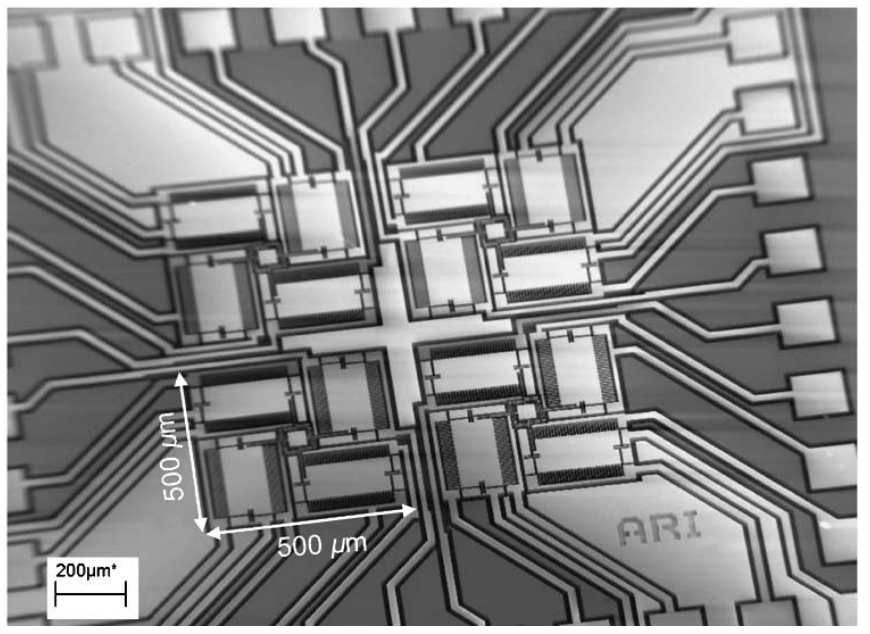

Fig. 10. SEM micrographs of fabricated and tested actuators for large arrays. (a) $0.8 \mathrm{~mm} \times 0.8 \mathrm{~mm}$ element with four unidirectional pure rotators. (b) $2 \times 2$ array for preliminary testing of $0.5 \mathrm{~mm} \times 0.5 \mathrm{~mm}$ elements with four bidirectional rotators.

parallel to "actuator 1" shuttle. The opposite arrangement gives negative angle direction. Analogous measurements were performed for the other two arrangements of actuators, again in both directions. As can be seen in the results of Fig. 9(a), the actuators were fabricated very uniformly such that all arrangements gave almost exactly the same optical deflections.

In common-mode arrangements, pistoning actuation of this device from $-30 \mu \mathrm{m}$ to $30 \mu \mathrm{m}$ has been observed as shown in Fig. 9(b): namely, if activating the up direction on all three actuators, their rotations combine as pure pistoning vertical motion of the micromirror. It should be noted that the different modes can be independently actuated and combined by activating proper electrodes with proper bias.

In addition to these larger devices, small actuators for high fill factor $(>96 \%)$ phased array applications are under development. We have fabricated and characterized square-layout tip-tilt-piston actuators as small as $0.8 \times 0.8 \mathrm{~mm}$ and down to $0.4 \times 0.4 \mathrm{~mm}$, shown in Figs. 10 and 12. For these applications, the actuators must be completely covered beneath the 
TABLE I

Measured Characteristics of Five Types of Devices: Triangle From Fig. 3 With INTEGRATED MirROR AND FOUR High FILl FACTOR ACTUATORS FOR MirROR BONDING

\begin{tabular}{l|r|r|r|r}
\hline & $\begin{array}{r}\text { X-axis scan } \\
\text { optical }^{\circ}\end{array}$ & $\begin{array}{r}\text { Y-axis scan } \\
\text { optical }^{\circ}\end{array}$ & $\begin{array}{r}\text { Res. freq no mirror } \\
\text { [Hz] }\end{array}$ & $\begin{array}{r}\text { Res. freq W/ mirror } \\
\text { [Hz] }\end{array}$ \\
\hline Triangle & -10 to 10 & -10 to 10 & n/a & 4096 \\
\hline HFF800 & 0 to 12.5 & 0 to 14.5 & 22727 & 1977 \\
\hline HFF600 & 0 to 16 & 0 to 16.1 & 13157 & $4000^{*}$ \\
\hline HFF500 & -22 to 22 & -23 to 16 & 11378 & 697.9 \\
\hline HFF400 & -12.5 to 14 & -15 to 6 & 64900 & $15500^{*}$ \\
\hline
\end{tabular}

HFF600 and HFF400 have not been characterized with bonded mirrors to date.

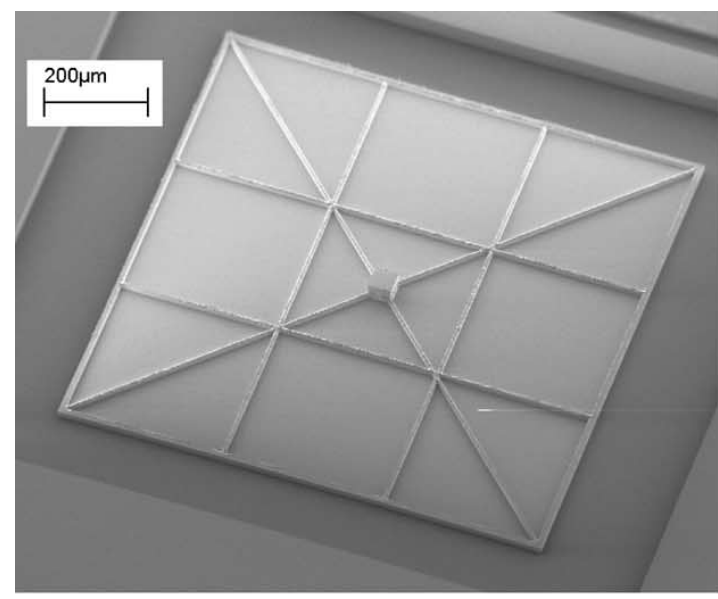

(a)

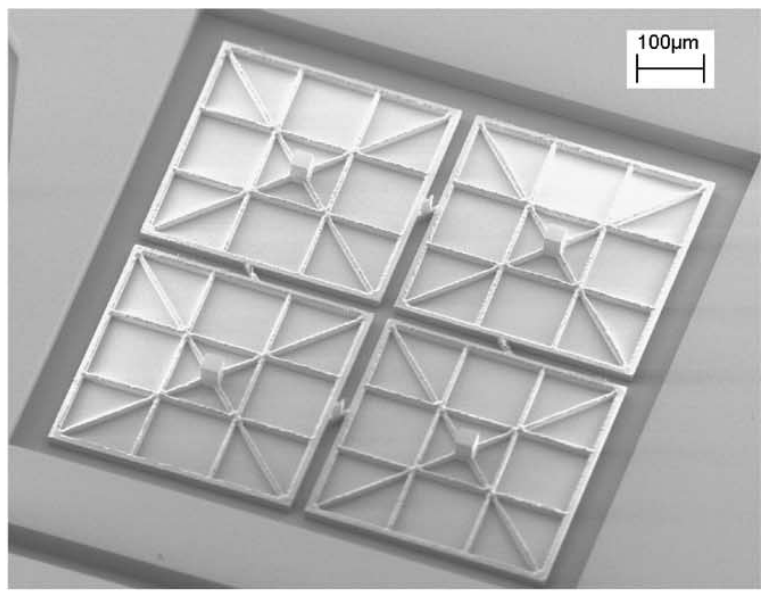

b)

Fig. 11. Fabricated low-inertial mirrors before release from the SOI wafer. Three thicknesses are visible: the thin mirror plate, thicker trusses for robust and flat support, and the tall pedestal.

micromirror's reflecting plate for high fill factor; therefore, additional fabrication/packaging solutions are required as discussed below in Section IV. Testing results to date are summarized in Table I. The smallest, $0.4-\mathrm{mm}$ actuators begin to push the process uniformity and critical dimensions limitations due to the limited aspect ratio of the DRIE etcher and due to undercutting in the etcher. We have found that such small devices have beam dimensions that, thus, become highly sensitive to any process variations. Large numbers of devices have been successfully tested as fabricated on a probe station, with a summary of the results given in Table I. The smallest device ( $400 \mu \mathrm{m}$ on a side) measured $>20^{\circ}$ of peak-to-peak optical deflection in both axes and $>64$ and $>30 \mathrm{kHz}$ resonant frequency in rotation and pistoning, respectively, prior to mirror bonding. A representative high field-factor actuator of $500 \mu \mathrm{m} \times 500 \mu \mathrm{m}$ dimensions (HFF500) achieves optical deflection angles beyond $-20^{\circ}$ to $20^{\circ}$ for both axes and greater than $-12-\mu \mathrm{m}$ to $12-\mu \mathrm{m}$ pistoning at $<80 \mathrm{~V}$ actuation.

\section{DESIGN FOR High FILl FACTOR}

\section{A. Fabrication of Low-Inertia Micromirrors}

Low-inertia micromirrors are fabricated in a separate SOI wafer employing a three-level selective DRIE process. The mirrors are realized from monolithic single-crystal silicon and consist of a full-height pedestal, thinned mirror plate, and midlevel trusses. The pedestal provides a bonding surface for attachment to the designed receiving platform on the actuators; it also serves as a standoff, providing separation between the mirror and actuator to allow sufficient rotation. Thinning of the mirror plate results in a significant reduction of the device's moment of inertia. Minimizing the inertia and mass of the structure allows higher resonant frequencies to be achieved without reducing the compliance of the actuation structure's suspension. The fabrication process also incorporates truss structures in order to provide a stiffening backbone for the thin mirror. Analytical and finite-element models (FEMs) of the device are employed to optimize the design of the mirror and truss structures in order to minimize the inertia as well as dynamic deformation of the mirror plate. Furthermore, the FEM is utilized to avoid undesirable, excitable modes. The trusses are also designed to allow the thin mirror plates to be metalized with approximately $100 \mathrm{~nm}$ of aluminum with minimal warping and static deformation.

The fabrication of the low-inertia micromirrors is initiated by growing a thick oxide layer $(\sim 2 \mu \mathrm{m})$ on an SOI wafer with a device layer thickness equal to the desired thickness of the pedestal plus the mirror plate. The oxide layer is then patterned with three consecutive lithography steps and RIE oxide etches. These etches define a multitiered oxide hard-mask for the subsequent DRIE silicon etches. During the first DRIE step, only the silicon that will be etched down to the buried oxide layer is exposed; the depth of this trench etch is greater than the desired thickness of the mirror plate. A blanket RIE oxide etch then completely removes the thinnest oxide hard mask while thinning the remaining oxide masks. The second DRIE determines the thickness of the trusses by recessing the mirror plate to the 
(a)

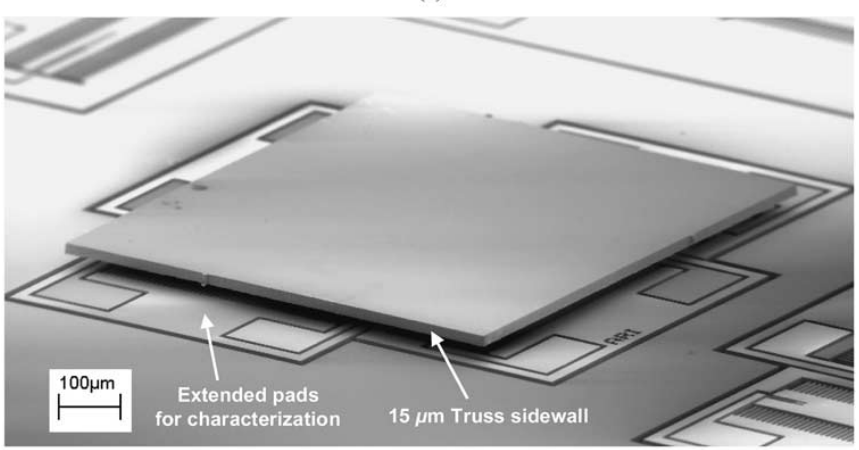

(b)

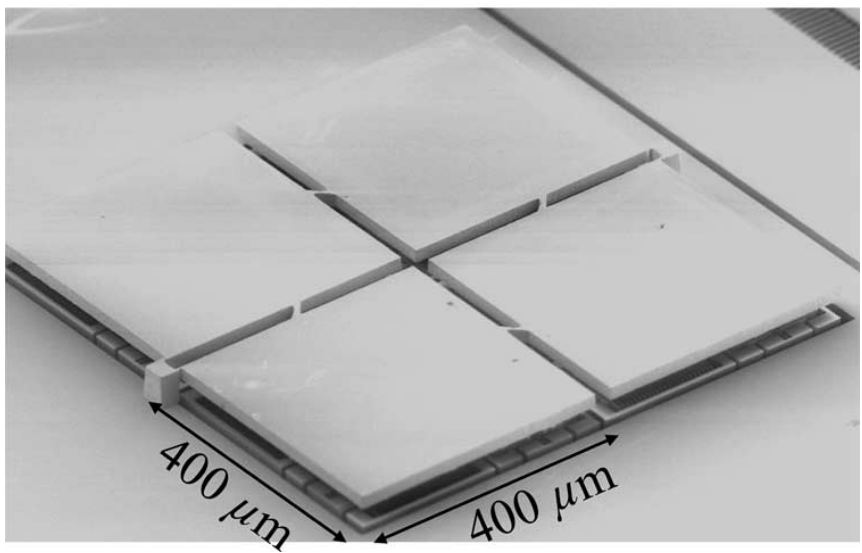

Fig. 12. SEM micrographs of fabricated high fill factor tip-tilt-piston micromirrors. (a) $0.8-\mathrm{mm} \times 0.8$-mm element with extended pads for electrical characterization, fully covered with a low-inertia micromirror. (b) $2 \times 2$ array of actuators with $0.4 \mathrm{~mm}$ on a side, with a $2 \times 2$ array of micromirrors batch transferred. The actuators have the same layout as shown in Fig. 10(b) and are appropriately scaled down. Tethers between micromirrors remain to be laser cut.

desired depth. A second oxide etch removes the truss hard mask. The final DRIE step lowers the parameter trench, truss beams, and mirror plate simultaneously; after the buried oxide layer is reached at the bottom of the outer trench, the etch is terminated when the desired mirror thickness is achieved. Finally, the mirrors are released in concentrated hydrofluoric acid (HF).

In this work, the thickness of the pedestal is $50 \mu \mathrm{m}$, the trusses are $15 \mu \mathrm{m}$ and the mirror thickness is $2 \mu \mathrm{m}$. SEMs of example fabricated mirror structures are presented in Fig. 11.

\section{B. Transfer and Bonding of Low-Inertia Micromirrors Onto Actuators}

The low-inertia micromirrors must be attached to the actuators, which are realized on a separate wafer. Transfer and bonding of individual thinned micromirrors onto the actuators was achieved using custom fabricated "capillary pickup fingers" [21], which hold the micromirror from the top, flat side. In this work, a small quantity of optical epoxy is applied to the actuator's pedestal platform using a micromanipulator and a simple tungsten probe tip. The mirror is held by the capillary pickup fingers on a separate micromanipulator. Following optical alignment of the mirror and actuator while viewing on the microscope, contact is made between the pedestal and platform. The surface forces tend to bring the pedestal into perfectly flat alignment with the stage as soon as contact is (a)

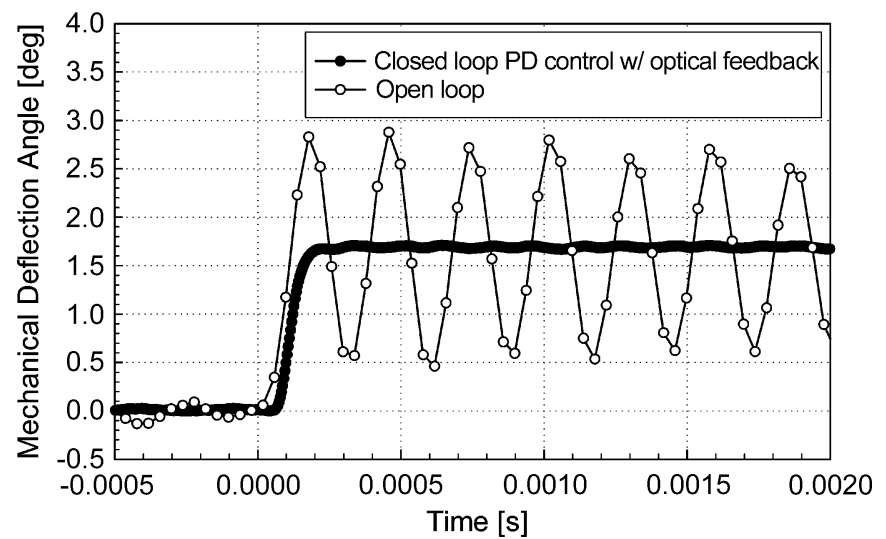

(b)

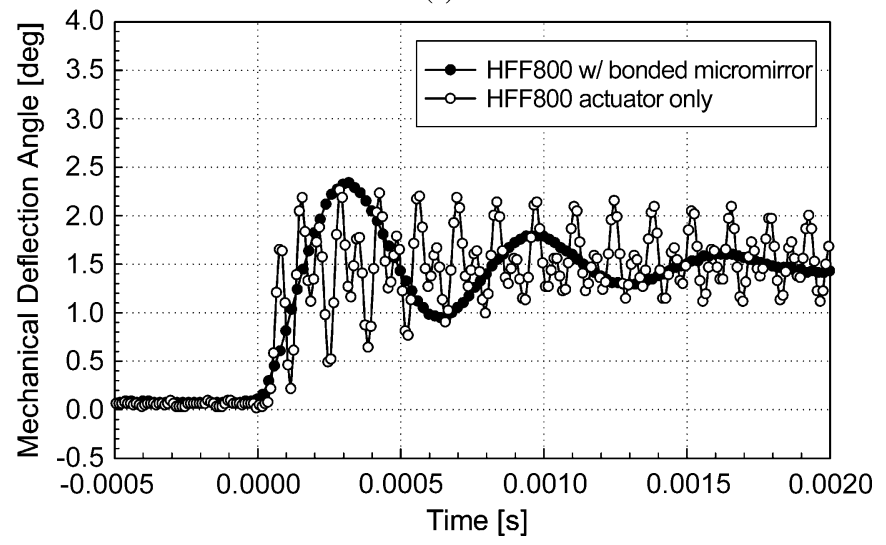

Fig. 13. Measured response of two different devices with efforts to decrease the step response settling time. (a) One-axis scanner has a $Q$ of nearly 100 and, therefore, settling times close to $100 \mathrm{~ms}$ in open loop actuation. Using optical feedback to sense device position and PD control, settling time is brought to $200 \mu \mathrm{s}$ from the input step. (b) For small array elements, high Q and multimode resonance result in long settling time without a bonded micromirror. With a bonded micromirror, squeeze film effect reduces $Q$ to 2.5 in this device, which then settles in $2 \mathrm{~ms}$ in an open-loop drive.

made, and the capillary pickup fingers then release the mirror. Finally, the chip is exposed to ultraviolet light followed by a curing step in a $125^{\circ} \mathrm{C}$ oven. Many devices were successfully assembled as shown in Fig. 12 and tested after micromirror transfer and bonding.

Batch bonding and alignment of multiple mirrors for largescale, high fill factor arrays is an ongoing effort, with the $2 \times 2$ array in Fig. 12(b) demonstrated to date.

It is important to note that for devices with bonded micromirrors the quality factor $(\mathrm{Q})$ (which is extracted from the measured step response) decreases significantly: namely, the devices in this work generally have very high Qs, on the order of 100, which makes their actuation in open-loop drive challenging. This is demonstrated in Fig. 13(a), with a one-axis scanner's measured step response. The scanner has a Q of nearly 100 at $\sim 3.5-\mathrm{kHz}$ resonance and, therefore, settling times close to $100 \mathrm{~ms}$ in open-loop actuation. The figure also shows our preliminary results on closed-loop control using optical feedback from a position-sensitive diode array to sense device position and proportional plus derivative (PD) control, bringing the $<1 \%$ settling time to $104 \mu$ s after the input step.

After the transfer and bonding of a low-inertia micromirror, the $\mathrm{Q}$ of the $800-\mu \mathrm{m}$ device is reduced from $\sim 50$ to 2.50 with a 
bonded micromirror, and for a 500- $\mu \mathrm{m}$ device, the final $\mathrm{Q}$ was 1.46. This reduction in $\mathrm{Q}$ is the result of increased damping due to the effects of surrounding air and is highly desirable for faster settling time operation. A settling time of $\sim 2 \mathrm{~ms}$ in open loop actuation was extracted from the plots shown in Fig. 13(b).

\section{CONCLUSION}

The present methodology is very promising for high speed and large static deflection 2-D scanning applications as well as high fill factor arrays of small mirrors with tip-tilt-piston capability for each individual element. It enables the designer to significantly optimize independent components and achieve desired overall performance. The actuators and mechanical linkages allow static two-axis rotation of micromirrors without need for gimbals or other specialized isolation methodology. The design presented utilizes a combination of actuators for making micromirrors capable of two-axis scanning as well as pistoning. Symmetry of the design allows both axes to have similar angular rotation and speed, which is highly desirable for applications such as vector or nonraster scanning. Smooth actuation of the device from onset greatly simplifies implementation of control systems.

Future goals include implementation of larger fully functional optical phased arrays of the demonstrated tip-tilt-piston elements with high fill factor.

\section{ACKNOWLEDGMENT}

The authors would like to thank M. Cohn, R. Roehnelt, and C. Keller for many useful technical discussions, as well as assistance with the micromirror transfer. The authors would also like to thank B. Cagdaser for helpful discussions and reading of the manuscript, and K. Castelino and M. Chang for discussions and assistance in closed-loop control measurements.

\section{REFERENCES}

[1] P. R. Patterson et al., "A MEMS 2-D scanner with bonded single-crystalline honeycomb micromirror," in Late News, Proc. Solid-State Sensor and Actuator Workshop, 2000, pp. 17-18.

[2] V. Milanovic, S. Kwon, and L. P. Lee, "Monolithic silicon micromirrors with large scanning angle," presented at the Optical MEMS'01, Okinawa, Japan.

[3] S. Blackstone et al., "SOI MEMS technologies for optical switching," presented at the Optical MEMS'01, Okinawa, Japan.

[4] R. Conant et al., "A flat high-frequency scanning micromirror," in Proc. Solid-State Sensor and Actuator Workshop, 2000, pp. 6-9.

[5] J. T. Nee et al., "Lightweight, optically flat micromirrors for fast beam steering," in Proc. 2000 IEEE/LEOS Int. Conf. Optical MEMS, pp. 9-10.

[6] U. Krishnamoorthy and O. Solgaard, "Self-aligned vertical comb-drive actuators for optical scanning micromirrors," in Proc. Optical MEMS '01, pp. 41-42.

[7] V. Milanovic, "Multilevel-beam SOI-MEMS fabrication and applications," J. Microelectromech. Syst., vol. 13, no. 1, pp. 19-30, Feb. 2004.

[8] V. Milanovic, S. Kwon, and L. P. Lee, "Monolithic vertical combdrive actuators for adaptive optics," in Proc. IEEE/LEOS Int. Conf. Optical MEMS, 2002, pp. 57-58.

[9] S. Kwon, V. Milanovic, and L. P. Lee, "Vertical microlens actuator for 3D imaging," presented at the Solid-State Sensor and Actuator Workshop, Hilton Head, SC, 2002.

[10] - "A high aspect ratio 2D gimbaled microscanner with large static rotation," in Proc. IEEE/LEOS Int. Conf. Optical MEMS, 2002, pp. 149-150.

[11] H. Schenk et al., "Large deflection micromechanical scanning mirrors for linear scans and pattern generation," IEEE J. Select. Topics Quantum Electron., vol. 6, pp. 715-722, Sep.-Oct. 2000.

[12] T. Brosnihan et al., "Embedded interconnect and electrical isolation for high-aspect-ratio, SOI inertial instruments," in Proc. IEEE Int. Conf. Solid-State Sensors and Actuators, vol. 1, 1997, pp. 637-640.
[13] V. Milanovic, G. Matus, T. Cheng, and B. Cagdasar, "Monolithic high aspect ratio two-axis optical scanner in SOI," in Proc. Int. Conf. Microelectromechanical Systems (MEMS 2003), pp. 255-258.

[14] F. Niklaus, S. Haasl, and G. Stemme, "Arrays of monocrystalline silicon micromirrors fabricated using CMOS compatible transfer bonding," $J$. Microelectromech. Syst., vol. 12, pp. 465-469, Aug. 2003.

[15] U. Srinivasan et al., "Fluidic self-assembly of micromirrors onto microactuators using capillary forces," IEEE J. Select. Topics Quantum Electron., vol. 8, pp. 4-11, Jan.-Feb. 2002.

[16] C. Rembe et al., "Stroboscopic interferometer with variable magnification to measure dynamics in an adaptive-optics micromirror," in Proc. IEEE/LEOS Int. Conf. Optical MEMS, 2000, pp. 73-74.

[17] J.-C. Tsai et al., "Analog micromirror arrays with orthogonal scanning directions for wavelength selective $1 \times N 2$ switches," in Proc. 12th IEEE Int. Conf. Solid-State Sensors, Actuators and Microsystems, 2003 , pp. 1776-1779.

[18] J.-L. A. Yeh et al., "Electrostatic model for an asymmetric combdrive," J. Microelectromech. Syst., vol. 9, pp. 126-135, Mar. 2000.

[19] J.-M. Kim, Y.-C. Ko, D.-H. Kong, J.-M. Kim, K. B. Lee, and D.-Y Jeon, "Fabrication of silicon optical scanner for laser display," in Proc. IEEE/LEOS Int. Conf. Optical MEMS, 21-24, 2000, pp. 13-14.

[20] J. M.-L. Tsai, H.-Y. Chu, J. Hsieh, and W. Fang, "The BELST II process for a silicon high-aspect-ratio micromaching vertical comb actuator and its applications," J. Micromech. Microeng., vol. 14, pp. 235-241, Nov. 2003.

[21] MEMS Precision Instruments [Online]. Available: http://www. memspi.com

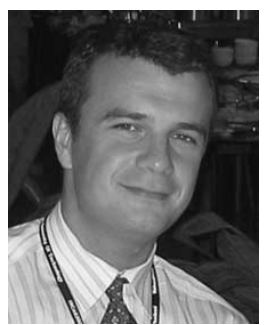

Veljko Milanović (S'92-M'99) received the M.Sc. and D.Sc. degrees in electrical engineering and $\mathrm{mi}-$ croelectronics from George Washington University, Washington, DC, in 1996 and 1998, respectively.

He was a Postdoctoral Researcher at the Berkeley Sensor and Actuator Center and a Guest Researcher in the Semiconductor Electronics Division of the National Institute of Standards and Technology. He founded the Adriatic Research Institute, Berkeley, $\mathrm{CA}$, with a focus on microsystems and nanosystems research and education for public benefit. He is also currently involved in biomolecular sciences research in the Nanoengineering Laboratory, Mechanical Engineering Department, University of California, Berkeley. He has been involved in microelectromechanical systems (MEMS) and nanotechnology research and development efforts for close to ten years.

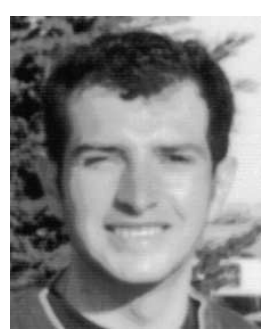

Gabriel A. Matus received the B.S. degree in materials science and engineering from the University of California, Berkeley, in 2000.

He was a MEMS Engineer and Researcher at the Berkeley Sensor and Actuator Center (BSAC) and a Process Engineer at Onix Microsystems, where he developed fabrication processes for optical switching systems. He is currently a Microsystems Researcher at the Adriatic Research Institute, Berkeley. The majority of his work relates to the design and fabrication of optical microsystems.

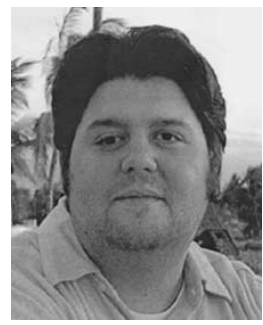

Daniel T. McCormick received the B.S.E. degree in biomedical engineering and electrical engineering from Duke University, Durham, NC, in 1999 and the M.S. and Ph.D. degrees in electrical and computer engineering from Cornell University, Ithaca, NY, in 2002 and 2003, respectively. His doctoral dissertation focused on microelectromechanical systems (MEMS) for in vivo optical coherence tomography imaging and radio frequency (RF) applications.

Since graduation, he has been a Postdoctoral Researcher in the Berkeley Sensor and Actuator Center at the University of California, Berkeley, continuing his research on the RF and biomedical applications of MEMS. He is also with the Adriatic Research Institute, Berkeley, where he is a Microsystems Engineer and a Course Instructor. His research interests also include integration of MEMS and microelectronics for high-precision instrumentation. 